\title{
Dispersion interaction in hydrogen-chain models
}

\author{
Ru-Fen Liu, ${ }^{1}$ János G. Ángyán, ${ }^{1}$ and John F. Dobson ${ }^{2}$ \\ 1) CRM2, Institut Jean Barriol, Nancy University and CNRS, \\ 54506 Vandoeuvre-lès-Nancy, France \\ 2) Queensland Micro and Nano Technology Centre, Griffith University, \\ 170 Kessels Road, Nathan, QLD 4111, Australia
}

(Dated: 11 February 2011)

We have investigated the dispersion interaction in hydrogen chain models via densityfunctional-theory-based symmetry-adapted perturbation theory (DFT-SAPT) using the asymptotically corrected PBE0 energy functional. The quasi-metallic and the insulating prototype systems were chosen to be hydrogen chains with equally- and alternately-spaced $\mathrm{H}_{2}$ units, respectively. The dependence of the dispersion energy on the chain length for quasi-metallic and insulating cases has been determined for two chains arranged either in pointing, or in parallel geometries. The results are compared with those previously calculated from a continuum coupled-plasmon approach [Phys. Rev. B, 77, 075436 (2008)]. The interaction energy has also been modeled by pairwise summations over short fragments of the chains, demonstrating the failure of the additivity principle for the quasi-metallic case, while confirming that the additivity is a qualitatively reasonable hypothesis for the insulating case. 


\section{INTRODUCTION}

The London dispersion interaction has its origin in the correlation of electron density fluctuations. Along with static multipole-multipole and induction terms, it dominates the attraction forces between two distant neutral systems. Its long-range and purely-correlation nature make it difficult to compute in any composite system, even in the absence of charge overlap between components. Typical examples include the stacking forces in layered materials like graphite $^{1}$ or boron nitride ${ }^{2}$, the formation of carbon nanotube bundles ${ }^{3-5}$, or the adhesive properties of gecko feet ${ }^{6}$. Remarkably strong aurophilic interactions in gold complexes $^{7,8}$ have been attributed also to London dispersion forces. In recent years much attention has been paid to the non-additivity of dispersion forces and to the way in which this effect depends on the metallic or non-metallic character of the system ${ }^{9,10}$.

For instance, considering two parallel one-dimensional (1D) wires, the conventional additivity principle, i.e. the sum of $R^{-6}$ contributions between two microscopic elements taken from each of the wires and separated by a distance $R$, leads to a standard $E \propto D^{-5}$ asymptotic behavior of the interaction energy with respect to the macroscopic interchain separation $D$. While this is in agreement with the results obtained by the random phase approximation (RPA) for two infinite insulating wires, for the metallic case RPA provides a different answer, predicting an asymptotic power law ${ }^{9,11}$ of the form $D^{-2}(\ln (K D))^{-3 / 2}$. The most credible theoretical calculations by Quantum Monte Carlo simulations recently reported similar behavior for parallel 1D homogeneous electron gases ${ }^{12}$. Misquitta et al. ${ }^{13}$ have recently investigated the power laws between two parallel linear wires with finite gaps using hydrogen chain model systems. Their results at intermediate interchain separations of quasi-metallic models approaches $D^{-2}$, close to the power law predicted for the true metallic case. Similar relationships have been found for the interaction of two-dimensional systems, like the zero-gap graphite where the power law predicted ${ }^{1,9,14,15}$ from the RPA energy goes to $D^{-3}$, in contradiction to the additive approach, which leads to an asymptotic power law of $D^{-4}$. These very distinct behaviors in asymptotic power laws between the metallic and insulating materials can be understood qualitatively by the fact that in metallic systems the electrons near the Fermi level are allowed to travel in the entire wire due to low-energy excitations, while such long-wavelength motions are usually prevented in insulating systems. The additive approach is unable to take into account these long-wavelength modes because 
it artificially confines electron motions in small fragments or domains of space and does not allow for a zero gap.

The consequences of a confinement of electrons to limited regions of space can be understood by analyzing the variation of the number of electrons associated with a subsystem, e.g. an atom, upon external electric field perturbation. The general framework for such an analysis of dispersion forces ${ }^{16-18}$ is based on the concept of distributed polarizabilities, introduced by A. J. Stone ${ }^{19}$. The lowest order distributed polarizability is the charge-flow polarizability, which tells the amount of charge displaced between two regions of the system in response to a unit difference in the electrostatic potential. In localized systems the charge-flow polarizability is expected to decay exponentially and becomes negligibly small for distant pairs of regions (atoms). In contrast, in strongly delocalized and metallic systems, where the electrons can almost freely travel, the charge flow between distant pairs of atoms or regions remains strong. Although the quantitative details of this behavior depend on the underlying partitioning scheme (e.g. space partitioning according to the topological analysis of the charge density, used in Refs. ${ }^{20}$ and ${ }^{16}$ or partitioning based on the assignment of density-fitting auxiliary functions to atoms used in Refs. ${ }^{17}$ ), the overall behavior is expected to be similar in both cases. A charge-flow to the nearest neighbor regions is equivalent to the buildup of an induced dipole extending over a pair of neighboring domains, therefore in localized systems we do not have to modify the picture of an essentially additive superposition of dipolar fluctuations leading to a $R^{-6}$ asymptotics: the only price to pay is a slight increase of the size of a fundamental unit from an atom to a pair of bonded atoms. In strongly delocalized or metallic systems the situation is quite different, since the buildup of fluctuating dipoles cannot be restricted to a small region of space; it can extend over large domains, being of a size comparable to the intersystem separation. In this latter case the charge-flow terms cannot be simply replaced by equivalent dipolar fluctuations and their explicit presence leads to a lowering of the asymptotic power law behavior, at least at intermediate distances. A mention of this phenomenon, predicted in Ref. ${ }^{9}$ and which was described in more detail in a very recent paper of Misquitta et al. ${ }^{13}$ and has also been briefly discussed by one of $\mathrm{us}^{21}$, appears for the first time in the literature in a particularly illuminating article by Longuet-Higgins and Salem in $1961^{22}$.

While several studies focused on the asymptotic behavior, White et al. ${ }^{23}$ considered the vdW interaction between two pointing wires, in the configuration represented in Fig.1(a) 
as a function of the length of the wire taken at a fixed distance of separation $D$. From continuum RPA calculations they showed that for the metallic case the dispersion interaction with respect to the increasing wire length is not only enhanced but also its rate of saturation becomes considerably slower, while for the insulating case the dispersion interaction energy gets saturated rapidly as the length of wire is increased beyond the separation distance. These findings can be considered as a strong indication that non-additivity effects are exhibited also in more complicated molecular systems, such as polyacetylene. Since the calculations in Ref. 23 were based on a highly simplified continuum model of the wires, it seemed to be relevant to examine whether the predictions hold for simple atomistic models going beyond a continuum approach. One of the simplest models that can be constructed is a system of two linear hydrogen chains. The hydrogen chain has been successfully used to model response and correlation properties in 1D systems ${ }^{13,24-29}$. Therefore we adopted the same model in an $a b$ initio approach and investigated the non-additivity of London dispersion effects in insulating and quasi-metallic hydrogen chain systems.

\section{COMPUTATIONAL DETAILS}

According intermolecular perturbation theory, the inter-molecular dispersion energy can be expressed as:

$$
E_{\mathrm{disp}}^{(2)}=-\sum_{a, b \neq 0} \frac{\left|\left\langle\phi_{a}^{A} \phi_{b}^{B}\left|\hat{V}^{A B}\right| \phi_{0}^{A} \phi_{0}^{B}\right\rangle\right|^{2}}{E_{a}^{A}-E_{0}^{A}+E_{b}^{B}-E_{0}^{B}}
$$

where $\left\{\phi_{a}^{A}, \phi_{b}^{B}\right\}$ are exact wave functions of the monomers $\mathrm{A}$ and $\mathrm{B}$ respectively, and $\hat{V}^{A B}$ is the operator of the mutual coulomb interaction between all charges (electrons and nuclei) in monomers A and B. By the virtue of the Casimir-Polder transformation Eq.(1) can be rewritten as a formula containing the product of two response functions of the monomers:

$$
E_{\text {disp }}^{(2)}=-\frac{1}{2 \pi} \int_{0}^{\infty} d \omega \int d r_{A} d r_{A}^{\prime} d r_{B} d r_{B}^{\prime} \frac{\chi^{A}\left(r_{A}, r_{A}^{\prime} ; i \omega\right) \chi^{B}\left(r_{B}, r_{B}^{\prime} ; i \omega\right)}{\left|r_{A}-r_{B}\right|\left|r_{A}^{\prime}-r_{B}^{\prime}\right|}
$$

The frequency dependent charge density response function, $\chi\left(r, r^{\prime} ; i \omega\right)$ can be calculated within the time-dependent HF theory, or in the time-dependent DFT (TDDFT) formalism, in which the important dynamical contributions are introduced by the exchange-correlation (xc) kernel $f_{x c}^{\mathrm{ALDA}}$ under the adiabatic local density approximation (ALDA). In order to take into account the antisymmetry of wavefunction, the latter method, i.e. the density functional 
theory based symmetry-adapted perturbation theory (DFT-SAPT or SAPT(DFT) $)^{30-36}$, was applied for the present studies. An advantage of the SAPT approach is that the dispersion contribution is clearly separated from other contributions such as electrostatic and induction interactions. Within quantum-chemical supermolecule techniques, these terms can be of similar magnitude as the dispersion term and it can be hard to separate them from each other. The monomer orbitals were determined by the Kohn-Sham method using the PBE0 functional with an asymptotic $1 / r$ correction potential (PBE0AC). The coupled dispersion energy (denoted as $E_{\text {disp }}^{c}$ hereafter) was obtained through solving the TDDFT equations. The uncoupled dispersion energy (denoted as $E_{\text {disp }}^{u}$ hereafter) was determined from the bare (uncoupled) Kohn-Sham (KS) polarizability, obtained from excitation energies and amplitudes corresponding to single electron excitations between KS orbitals; i.e. neglecting all screening effects in the monomer response functions. All calculations were performed with the Gaussian-type double zeta basis set (vdz) by MOLPRO 2008 program ${ }^{37}$. Since in this work we focus mainly on the effects to the polarizability components parallel to the hydrogen chain, such a relatively small basis set seems to be appropriate. Contributions from the perpendicular polarizability components, which are underestimated in the vdz basis, are essentially irrelevant to understand the qualitative behavior of the dispersion interaction with increasing chain length.

The geometry of a linear hydrogen chain can be described by two internal parameters: the bond length of the $\mathrm{H}_{2}$ unit ( $r$ in Fig.1(a)) and the distortion parameter $\kappa \equiv R / r$. Hence, for $\kappa>1$ an infinite chain has a lattice constant $a \equiv(1+\kappa) r$. The equilibrium distance $r$ for an infinite equally-spaced $(\kappa=1)$ hydrogen chain had been studied at the near Hartree-Fock limit $^{38}$ and later by the second and third order Møller-Plesset perturbation theory ${ }^{39,40}$. These works reported the distance to be around $1.84 a_{0}$, which was utilized in the present work. Hydrogen chains $\left(\mathrm{H}_{2}\right)_{n}$ with length parameter up to $n=2 \sim 25$ were constructed. TableI presents the HOMO-LUMO gaps as a function of increasing chain length $n$ for different values of $\kappa$. The gap decreases significantly when the chain length (denoted as $L(n)$ hereafter) is increasing for the equally-spaced $(\kappa=1)$ hydrogen chain. For instance, there is an about $70 \%$ reduction of the gap from $n=2$ to $n=10$ for $\kappa=1$, while only $15 \%$ for $\kappa=2$, i.e. for the alternately-spaced hydrogen chain with maximal distortion considered in this study.

One can also discriminate between metallic and insulating systems by evaluating the fluctuation of electron position via the so-called electron localization tensor ${ }^{41-44}$. The trace 
of the tensor is the Resta localization index (denoted as $\left\langle r^{2}\right\rangle_{c}$ hereafter), i.e. the densityweighted second cumulant moment per electron of the exchange-correlation hole $\left(h_{x c}\right)$ :

$$
\begin{aligned}
\left\langle r^{2}\right\rangle_{c} \equiv \operatorname{Tr}\left(\left\langle\hat{r}_{\alpha} \hat{r}_{\beta}\right\rangle_{c}\right)=-\frac{1}{2 N} \operatorname{Tr}\left(\int d \mathbf{r} \rho(\mathbf{r})\right. & \left.\int d \mathbf{r}^{\prime}\left(r-r^{\prime}\right)_{\alpha}\left(r-r^{\prime}\right)_{\beta} h_{x c}\left(\mathbf{r}, \mathbf{r}^{\prime}\right)\right) \\
& =-\frac{1}{2 N} \int d \mathbf{r} \rho(\mathbf{r}) \int d \mathbf{r}^{\prime}\left(r-r^{\prime}\right)^{2} h_{x c}\left(\mathbf{r}, \mathbf{r}^{\prime}\right) .
\end{aligned}
$$

A larger value of this index indicates a more delocalized nature for the electrons in the system. Fig.2 presents $\left\langle r^{2}\right\rangle_{c}$ per length calculated from a single-determinant wave-function for chain length $n=25$. We fixed the bond length $r$ of $\mathrm{H}_{2}$ and evaluated $\left\langle r^{2}\right\rangle_{c}$ per length as a function of the distortion parameter $\kappa$ for the alternately-spaced hydrogen chains (blue circles); whereas for the equally-placed ones (i.e. fixed $\kappa=1$, red diamonds in the insert), $\left\langle r^{2}\right\rangle_{c}$ per length was computed as a function of $r$. Because the equally-spaced (alternatelyspaced) chain with large $r(\kappa)$ effectively consists of isolated $\mathrm{H}$ atoms $\left(\mathrm{H}_{2}\right.$ molecules), the calculated $\left\langle r^{2}\right\rangle_{c}$ per length is decreasing. However, $\left\langle r^{2}\right\rangle_{c}$ per length for $\kappa=1$ displays much stronger fluctuation in electron position compared to that with the same $r$ but $\kappa>1$, i.e. $\left\langle r^{2}\right\rangle_{c}$ per length decreases sharply once the distortion is introduced. Besides, we found that the calculated $\left\langle r^{2}\right\rangle_{c}$ per length for alternately-spaced hydrogen chains does not depend strongly on $r$ as one can see similar behavior for the cases with $r=1.84 a_{0}$ and $1.44 a_{0}$ in the figure. Hence, in the large $n$ limit, the chain with $\kappa=1$, i.e. the equally-spaced hydrogen chain, could be considered as a quasi-metallic one-dimensional system, while that with $\kappa>1$, i.e. the alternately-spaced one, is a good model for an insulating system.

Note that we are interested in the dispersion interaction at an inter-chain separation distance $D$ large enough compared to $r$, while smaller than the asymptotic distance for the interaction between the entire finite chains. Therefore the interchain separation distance $D$ has been chosen as $8 a_{0}$ for both the parallel and the pointing geometries.

\section{RESULTS}

\section{A. Pointing geometry}

Fig.3 presents the total DFT-SAPT dispersion energies $E_{\text {disp }}^{c}$ for the pointing configuration. These results agree with previous observations from continuum RPA models ${ }^{23}: 1$ ) the rapid saturation of $E_{\text {disp }}^{c}$ with large $L(n)$ occurs only for insulating chains ( $\kappa>1$, circles); 2$)$ 
comparing with a rather small-gap system, e.g. $\kappa=1.25$, there is a very distinct behavior of $E_{d i s p}^{c}(n)$ for the metallic case $(\kappa=1)$, i.e. a continuously increasing vdW energy that appears to saturate only very slowly with increasing $n$. The specific character of the saturation in $E_{d i s p}^{c}$ with respect to $L(n)$ for insulating cases is consistent with pairwise additivity, as one can formulate the pairwise summation:

$$
E_{\mathrm{disp}}^{\mathrm{add}}=-\sum_{i, j} \frac{A}{R_{i j}^{6}}
$$

where $A$ is the dispersion coefficient between pairs $\{i, j\}$. It can be shown that the $n$ dependence of $E_{\text {pointing }}^{\text {add }}(n)$ within this additive pairwise summation model ${ }^{45}$ gives $\Delta E_{\text {pointing }}^{\text {add }} \propto$ $n^{-4}$ (See Appendix A.). It is then interesting to see whether the full non-additive DFTSAPT treatment agrees with the additive pairwise result for the most strongly insulating cases, i.e. those with larger $\kappa$. To confirm this behavior, we tried a fit consistent with Eq.(A10), i.e.

$$
E_{\text {pointing }}^{\mathrm{SAPT}, \mathrm{fit}}(n)=A+B\left\{\frac{1}{\left(\frac{1+\xi}{b}+n\right)^{p}}-\frac{1}{32\left(\frac{1+\xi}{2 b}+n\right)^{p}}\right\}
$$

where $A, B$ and $p$ are independent of $n$. Here, since the DFT-SAPT energies for $\kappa=2$ saturate very rapidly with increasing $n$, we used only the $2 \leq n \leq 10$ data. The results are shown in TableII. Clearly, the DFT-SAPT exponent $p$ is approaching 4 for the larger $\kappa$ values, in rough agreement with the prediction of the additive model as given in Appendix A.

However, for the metallic case ( $\kappa=1$, diamonds) the dispersion energy saturates much more slowly with $n$. Thus, for this case it is meaningful to use all the data $(n=2 \sim 25)$ for fitting the $n$-dependence. We tried a power law of form $E_{d i s p}=A+B n^{-p}$, but did not achieve a very good fit. Inspired by the logarithmic factors that occur in the analytic theory of vdW attraction between infinitely long conducting parallel wires ${ }^{9,12}$, we tried to fit the $n$-dependence via the function

$$
E_{\text {pointing, }, \kappa=1}^{\mathrm{SAPT}, \mathrm{fit}}(n)=A+B n^{-p} \ln (n)
$$

With this ansatz we have obtained a robust fit and the exponent $p$ was of order 1 (see TableII). This fit suggests that the energy in pointing geometry does indeed saturate to a finite value for large $n$, but only quite slowly. The empirical form of Eq.(6) seems to be the first attempt in the literature to provide an analytic prediction for the $n$-dependence in 
non-additive models such as DFT-SAPT. It would be interesting to find the corresponding analytic theory. However, in view of the necessity to solve spatial integral equations without translational symmetry, the corresponding mathematics is probably quite heavy, leading to equations at least as complex as the Wiener-Hopf formalism ${ }^{46,47}$.

In the insert, the uncoupled $E_{d i s p}^{u}$ is given for comparison to $E_{d i s p}^{c}$, both for the metallic case. It is shown that $E_{d i s p}^{u}$, which is closely related to MBPT2 (second order many-body theory) correlation energy, results in a very rapidly increasing dispersion energy with $n$. This is due to the underestimation of excitation energies when these are determined directly from the bare energy differences of KS orbitals. On the other hand, the coupled $E_{d i s p}^{c}$ shows a moderate increase in magnitude since the quasi-particle excitation energies are calculated under the strong screening effect for the metallic system.

\section{B. Parallel geometry}

Fig.4 presents the DFT-SAPT dispersion energy per $\mathrm{H}_{2}$ unit (denoted as $\epsilon_{\text {disp }}^{c}$ hereafter) for parallel geometry (Fig.1(b)). The saturation when $L(n)>D$ is significantly slower with $n$ than that in the pointing case. This behavior can be further confirmed through the $\mathrm{H}_{2}$ pairwise summation of the dispersion energy (Eq.(4)). The $n$-dependence of $\epsilon_{\text {disp }}^{c}$ was found to be $\Delta \epsilon_{\text {parallel }}^{\text {add }}(n) \propto n^{-1}$ when $n$ is large ${ }^{48}$. We tried a fitting function with the following form based on Salem's work ${ }^{48}$ (see Appendix B):

$$
\epsilon_{\text {parallel }}^{\mathrm{SAPT}, \mathrm{fit}}(n)=A+B \frac{n^{p}}{\left(1+(b n)^{2}\right)^{2}}+C \tan ^{-1}(b n)
$$

and the numerical fit results are given in TableIII. Here, the exponent $p$ is expected to approach the value of 3 for systems with larger $\kappa$. Our fit results suggest a similar trend, i.e. the additive nature would become stronger for larger $\kappa$. Note that, the robust fits by employing this simplified function are obtained with the data set $n=7 \sim 25$ for $\kappa=2,1.5$, while the whole data set was used for $\kappa=1.25$ so that the slower saturation rate for this case could be taken into account. However, Eq.(7) is not able to give a good fit for the metallic case, so we have adopted the empirical form of Eq.(6). The exponent $p$ has been found to be of the order of 1 . Since analytic RPA calculations ${ }^{9}$ for infinite parallel wires show a finite energy per unit length regardless of metallic or insulating character, it seems likely that the present approach would show a saturation to a finite energy per $\mathrm{H}_{2}$ unit at 
infinite length even in the metallic case. Clearly, however, the largest $n$ values studied here are not yet in this asymptotic region of very long wires for the metallic case.

Furthermore, to address the non-additivity effect clearly, the additive pairwise summation approach was applied by using the DFT-SAPT method for the present hydrogen chain studies. DFT-SAPT calculations have been performed for the dispersion energy between smaller fragments taken from the two chains. In particular, we have considered (1) all possible pairs of two $\mathrm{H}_{2}$ units taken from each chain, and (2) pairs of two $\mathrm{H}_{4}$ units taken from each chain, and summed up dispersion energies (denoted as $\epsilon_{\text {disp }}^{c, p a i r}$ hereafter) over all possible pairs. In this case, we considered only $\kappa=2$ (the strongest insulating system considered here) and $\kappa=1$ (metallic). The results are presented in Fig. 4: open triangles stand for $\kappa=2$ and crosses for $\kappa=1$. First of all, if the additive approach is valid for insulating systems, one should expect that the result from pairwise summation of pair DFT-SAPT dispersion energies should agree with full DFT-SAPT calculations for $\kappa=2$. Although the result presented in Fig. 4 (open triangles) gives reasonable interaction energies, they are still inaccurate compared with $\epsilon_{\text {disp }}^{c}$ calculated for the entire system (dark blue dots). This small but significant discrepancy is attributed to the long-ranged contributions in coulomb screening which are only taken into account in calculations for entire chains, but which are missing from the additive approach ${ }^{10,49}$. Therefore, we have concluded that in a strict sense, even for insulating systems, the additive approach is only qualitatively valid.

On the other hand, for $\kappa=1, \epsilon_{d i s p, \mathrm{H}_{2}}^{\mathrm{c} \text {,pair }}$ (thin crosses) gives a qualitatively wrong behavior when compared to $\epsilon_{d i s p}^{c}$ (red dots). This can be understood by the fact that the non-additive effects due to the screening for $\kappa=1$ lead to an $n$-dependent $\epsilon_{\text {disp }}(n)$, which corresponds not only to a quantitatively much stronger interaction strength, but which displays also a qualitatively very different behavior at large $n$. Note that $\epsilon_{d i s p, \mathrm{H}_{2}}^{\mathrm{c}, \mathrm{pair}}$ leads to a saturation of the energy at large $n$ regardless of the value $\kappa$. The loss of correlation could not be retrieved even if one increased the size of the fragment, e.g. by passing from the $\mathrm{H}_{2}$ to the $\mathrm{H}_{4}$ unit (dashed line with thick crosses). A similarly strong non-additivity has been observed in the case of two metallic chains in the pointing geometry, where our attempts to reproduce the full length results as a sum of contributions coming from pairs of $\mathrm{H}_{2}$ or $\mathrm{H}_{4}$ units fail completely (c.f. red crosses in FIG.3). These results indicate that probably other partitioning schemes serving as a basis of an additive approach would fail also to account for dispersion forces in low-dimensional metallic systems. 


\section{CONCLUSION AND DISCUSSION}

We have computed the dispersion interaction energy $\epsilon_{\text {disp }}$ between two "wires" made up of hydrogen atoms. For equally spaced hydrogens, these wires mimic a metal, with an electronic band gap that goes to zero as the length goes to infinity. However, with unequally spaced hydrogens, we were able to model an insulator where the finite gap becomes stable as the length increases. To calculate $\epsilon_{d i s p}$, we have used second-order Symmetry-Adapted Perturbation Theory, and treated both parallel and pointing geometries (see Fig.1) of the two wires. Unlike previous works on finite atomic chains, our calculations were focused mainly on the dependence of $\epsilon_{d i s p}$ on the length of the wires. We found that, in both parallel and pointing geometries, the length dependence of $\epsilon_{\text {disp }}$ differed markedly between the "metallic" and "insulating" cases. In particular the energy continued to increase with length (up to $n=25$ monomers) for the metallic case but saturated for relatively small $n$ value in the insulating cases. Explicit calculation showed that the dispersion interaction energy obtained by a pairwise-summation approach is qualitatively unsatisfactory in the metallic case, and it severely underestimates the dispersion energy as the wire length increases.

For insulating cases the pair-wise additive approach remains reasonable although it becomes qualitatively inaccurate for small band-gap systems. For the case of wires in the pointing geometry, our discrete-atom work lends credence to the only previous work on this problem $^{23}$, which was done in a much simpler continuum model and had some possible issues with the treatment of end effects. Our work raises an interesting question in the case of pointing geometry, namely whether the total dispersion energy saturates with increasing length. It is clear from our results that this is so for the insulating case. Neither our present calculations, nor previous works with a continuum model ${ }^{23}$ were able to resolve this issue in the metallic case, because of the computational limitations on the length of wire that could be modeled numerically. It seems that a tractable analytical model may be necessary in order to resolve this issue definitely. Our $n^{-1.28} \ln (n)$ fit, if it continued to be valid as $n$ increased, would definitively show that there is a finite dispersion energy as $n$ goes to infinity, since $n^{-|p|} \ln (n)$ approaches zero as $n$ approaches infinity for any nonzero $p$.

After the present work was commenced, we became aware of the work of Misquitta et al. ${ }^{13}$, which treats parallel hydrogen chains with a finite energy gap. Our results agree with that work where applicable. In addition, here we have also considered chains of atoms in the 
collinear "pointing" configuration and in general we mainly focus on the unusual dependence of the interaction on the length of the chain.

\section{ACKNOWLEDGMENTS}

This work has been partly financed by the French Agence Nationale de la Recherche (ANR) under the contract ANR07BLAN-0272 (Project WADEMECOM) and by the FrenchAustralian Scientific Project FAST. JFD acknowledges ARC Discovery Grant DP1096240. RFL specially thanks Prof. Georg Jansen for helpful discussions on the DFT-SAPT method.

\section{Appendix A: The additive pairwise summation approach: pointing geometry}

The dispersion interaction between two parallel and two circular chains separated at the intermediate distance had been investigated by Salem ${ }^{48}$, and that between two pointing rods by De Rocco et al. ${ }^{45}$. Both studies concentrated on the total dispersion energy under the assumption of additivity and the orientation-dependence between subelement pairs was neglected, i.e. they treated the $C_{6}$ coefficients as isotropic for each subelement. The validity of these two assumptions had been discussed by Zwanzig ${ }^{49}$, who showed a fivefold underestimation of the total dispersion energy due to ignoring the interaction between small fragments in the chain (effect of non-additivity), and a fourfold overestimation due to the neglect of anisotropic polarizability of the fragments. Since the purpose of this Appendix is to provide working equations for the additive approach, no attempt will be made to include non-additive effects in these analytical equations. The basic subunit will be a $\mathrm{H}_{2}$ fragment cut out of the chain.

In a multipole expansion of the dispersion energy, the leading term, which depends on the $\mathrm{C}_{6}$ dispersion coefficients of the fragments, is due to the interaction of the fluctuating dipoles and can be written as

$$
E_{d i s p}=-\frac{1}{2 \pi} \int_{0}^{\infty} d u T r\left[\alpha_{\alpha \beta}^{A}(i u) T_{\beta \delta} \alpha_{\delta \gamma}^{B}(i u) T_{\gamma \alpha}\right]
$$

where $T_{\alpha \beta}$ is the dipole-dipole interaction tensor:

$$
T_{\alpha \beta}=\frac{1}{R^{5}}\left\{3 R_{\alpha} R_{\beta}-R^{2} \delta_{\alpha \beta}\right\}
$$


and $\alpha(i u)$ is the frequency dependent polarizability of the object. Here, we will use the above formula to construct the sum of dispersion energy over the $\mathrm{H}_{2}$ pairs for two chains in pointing and parallel geometries.

\section{1. $E_{\text {pointing }}^{H_{2}}$ from $\mathbf{H}_{2}$-pair summation}

The anisotropic polarizability of $\mathrm{H}_{2}$ in the matrix representation is given as:

$$
\alpha^{H_{2}}(i u)=\left(\begin{array}{ccc}
\alpha_{\perp}(i u) & 0 & 0 \\
0 & \alpha_{\perp}(i u) & 0 \\
0 & 0 & \alpha_{\|}(i u)
\end{array}\right)
$$

For the pointing geometry (see Fig.1(a)), the position of $\mathrm{H}_{2}$ units in $\mathrm{A}(\mathrm{B})$ (chosen from the middle of the $\mathrm{H}-\mathrm{H}$ bond) is $\mathbf{r}^{A(B)}=\left(0,0, z_{A(B)}\right)$. Thus the distance vector between two $\mathrm{H}_{2}$ is

$$
\mathbf{R}=\mathbf{r}^{B}-\mathbf{r}^{A}=\left(0,0, z_{B}-z_{A}\right) \equiv(0,0, z)
$$

The interaction operator $T_{\text {pointing }}$ can be constructed directly as $T_{\text {pointing }} \equiv \frac{\mathrm{t}_{\text {pointing }}}{z^{3}}$ :

$$
\mathrm{t}_{\text {pointing }}=\left(\begin{array}{rrr}
-1 & 0 & 0 \\
0 & -1 & 0 \\
0 & 0 & 2
\end{array}\right)
$$

Substituting $\alpha^{H_{2}}(i u)$ and $T_{\text {pointing }}$ into Eq.(A1) we find the dispersion energy between two pointing $\mathrm{H}_{2}$ molecules to be

$$
\varepsilon_{\text {pointing }}^{H_{2}}=-\frac{2 C_{\perp \perp}+4 C_{\|\|}}{z^{6}}
$$

where

$$
\begin{aligned}
C_{\perp \perp} & \equiv \frac{1}{2 \pi} \int_{0}^{\infty} d u \alpha_{\perp}(i u) \alpha_{\perp}(i u) \\
C_{\|\|} & \equiv \frac{1}{2 \pi} \int_{0}^{\infty} d u \alpha_{\|}(i u) \alpha_{\|}(i u)
\end{aligned}
$$

For each $\mathrm{H}_{2}$ pair, say the $s^{t h} \mathrm{H}_{2}$ in $\mathrm{A}$, and the $t^{t h} \mathrm{H}_{2}$ in $\mathrm{B}$ (see the labels with gray color in Fig.1), the general expression for $\mathbf{R}_{s t}$ is

$$
\mathbf{R}_{s t}=D \mathbf{r}_{s t}=D(0,0,1+\xi+b(s+t))
$$


i.e. $z_{s t}=D(1+\xi+b(s+t))$, where $b \equiv \frac{a}{D}$ and $\xi \equiv \frac{r}{D}$. $a$ is the lattice constant, $D$ is the distance between two ends of $\mathrm{A}$ and $\mathrm{B}$ chains, and $r$ is the bond length of $\mathrm{H}_{2}$. By summing all $\mathrm{H}_{2}$ pairs, we have

$$
E_{\text {pointing }}^{H_{2}}(n)=-\frac{2 C_{\perp \perp}+4 C_{\|\|}}{D^{6}(1+\xi)^{6}} F\left(\frac{b}{1+\xi}, n\right)
$$

where

$$
F(b, n) \equiv \sum_{s=0}^{n-1} \sum_{t=0}^{n-1} \frac{1}{(1+b(s+t))^{6}}
$$

\section{2. $n$-dependence of $E_{\text {pointing }}^{\mathrm{H}_{2}}(n)$}

When the chains are widely separated, i.e. $D \gg a$ and $b \ll 1$, one can replace $s$ and $t$ summations by integrations in Eq.(A11), yielding a closed result:

$$
\begin{aligned}
& F(b, n) \approx G(b, n) \equiv \int_{0}^{n} d s \int_{0}^{n} d t \frac{1}{(1+b(s+t))^{6}} \\
&=\frac{1}{20 b^{2}}\left[1-\frac{2}{(1+b n)^{4}}+\frac{1}{(1+2 b n)^{4}}\right]
\end{aligned}
$$

Substituting Eq. (A12) into Eq. (A10) and taking $n \rightarrow \infty$, we obtain

$$
E_{\text {pointing }}^{H_{2}}(\infty)=-\frac{C_{6, \text { pointing }}^{H_{2}}}{20 b^{2} D^{6}(1+\xi)^{6}}
$$

where $C_{6, \text { pointing }}^{H_{2}} \equiv 2 C_{\perp \perp}+4 C_{\|\| \|}$. Since $b=D / a$, (A13) implies that

$$
E_{\text {pointing }}^{\text {add }} \propto D^{-4} \quad \text { when } \quad D \gg a \quad \text { and } \quad L \ll D
$$

However, here it is the dependence on $L(n)$ that is of interest, and our numerical results calculated by DFT-SAPT method were determined a fixed interchain separation $D=8 a_{0}$ which is not large. Thus the continuum approximation may not be very accurate for the total dispersion energy. Nevertheless in evaluating the asymptotic difference between dispersion energies for $n \rightarrow \infty$ and finite $n$, we can still reasonably replace sums by integrals, so that

$$
F(b, n)-F(b, \infty) \quad \approx \quad-\frac{1}{20 b^{2}}\left[\frac{2}{(1+b n)^{4}}-\frac{1}{(1+2 b n)^{4}}\right]
$$


That is, for sufficiently large $n$ and fixed $D$,

$$
\begin{aligned}
& E_{\text {pointing }}^{H_{2}}(n)-E_{\text {pointing }}^{H_{2}}(\infty) \\
\approx & -\frac{C_{6, \text { pointing }}^{H_{2}}}{D^{6}(1+\xi)^{6}}\left\{F\left(\frac{b}{1+\xi}, n\right)-F\left(\frac{b}{1+\xi}, \infty\right)\right\} \\
= & \frac{C_{6, \text { pointing }}^{H_{2}}}{10 D^{6} b^{6}}\left\{\frac{1}{\left(\frac{1+\xi}{b}+n\right)^{4}}-\frac{1}{32\left(\frac{1+\xi}{2 b}+n\right)^{4}}\right\}
\end{aligned}
$$

¿From this it is clear that the length dependence within the additive pairwise model is

$\Delta E^{a d d} \propto n^{-4} \propto L(n)^{-4}$. This result is consistent with the previous findings concerning the dispersion interaction between two collinear rods ${ }^{45}$.

\section{Appendix B: The additive pairwise summation approach: parallel geometry}

\section{1. $E_{\text {parallel }}^{H_{2}}$ from $\mathbf{H}_{2}$-pair summation}

In the parallel geometry, let the $\mathrm{H}_{2}$ in $\mathrm{A}$ align along $\mathbf{r}^{A}=\left(0,0, z_{A}\right)$, and that in $\mathrm{B}$ align along $\mathbf{r}^{B}=\left(D, 0, z_{B}\right)$, then the distance between the two is given as:

$$
\mathbf{R}=\mathbf{r}^{B}-\mathbf{r}^{A} \equiv(D, 0, z) \equiv D(1,0, \zeta)
$$

where $z=z_{B}-z_{A}$ and $\zeta \equiv \frac{z}{D}$. One obtains $T_{\text {parallel }} \equiv \frac{\mathrm{t}_{\text {parallel }}}{D^{3}}$ :

$$
t_{\text {parallel }} \quad=\frac{1}{\left(1+\zeta^{2}\right)^{5 / 2}}\left(\begin{array}{ccc}
2-\zeta^{2} & 0 & 3 \zeta \\
0 & -1-\zeta^{2} & 0 \\
3 \zeta & 0 & -1+2 \zeta^{2}
\end{array}\right) .
$$

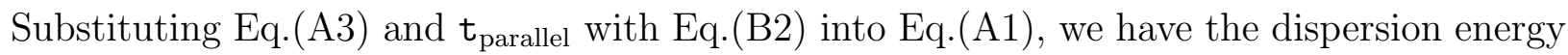
between two parallel (but not necessarily in registry, i.e. $\zeta$ can be nonzero) $\mathrm{H}_{2}$ chains as:

$$
\begin{aligned}
\varepsilon_{\text {parallel }}^{H_{2}}= & -\frac{1}{D^{6}\left(1+\zeta^{2}\right)^{5}}\left\{\left(1-2 \zeta^{2}\right)^{2} C_{\|\|}+18 \zeta^{2} C_{\perp \|}\right. \\
& \left.+\left(5-2 \zeta^{2}+2 \zeta^{4}\right) C_{\perp \perp}\right\} \\
= & -\frac{1}{D^{6} r^{10}}\left\{9\left(C_{\perp \perp}-2 C_{\perp \|}+C_{\|\| \|}\right)\right. \\
& -6\left(C_{\perp \perp}-3 C_{\perp \|}+2 C_{\|\|}\right) r^{2} \\
& \left.+2\left(C_{\perp \perp}+2 C_{\|\| \|}\right) r^{4}\right\}
\end{aligned}
$$


where

$$
C_{\perp \|} \equiv \frac{1}{2 \pi} \int_{0}^{\infty} d u \alpha_{\perp}(i u) \alpha_{\|}(i u)
$$

and $r^{2} \equiv 1+\zeta^{2}$. The distance for a given $\mathrm{H}_{2}$-pair $\mathbf{R}_{\mathbf{s t}}$ is

$$
\mathbf{R}_{s t}=D\left(1,0, \zeta_{s t}\right)=D(1,0,(t-s) b)
$$

Note that the summations of $s$ and $t$ can be further reduced by one with an extra factor $2(n-m)$ and the term to be subtracted due to the double counting when $s=t$. Therefore, by summing all $\mathrm{H}_{2}$ pairs, the pairwise summation of dispersion energy per length $\mathrm{H}_{2}$, i.e. $\epsilon_{\text {parallel }}^{H_{2}} \equiv \frac{E_{\text {parallel }}^{H_{2}}}{n}$, is given as:

$$
\begin{aligned}
\epsilon_{\text {parallel }}^{H_{2}}(n)= & -\frac{1}{D^{6}}\left\{9\left(C_{\perp \perp}-2 C_{\perp \|}+C_{\|\| \|}\right) \frac{H_{5}(b, n)}{n}\right. \\
& -6\left(C_{\perp \perp}-3 C_{\perp \|}+2 C_{\|\|}\right) \frac{H_{4}(b, n)}{n} \\
& +2\left(C_{\perp \perp}+2 C_{\|\| \|}\right) \frac{H_{3}(b, n)}{n} \\
& \left.-\left(5 C_{\perp \perp}+C_{\|\| \|}\right)\right\},
\end{aligned}
$$

where

$$
H_{\lambda}(b, n)=\sum_{m=0}^{n} \frac{2(n-m)}{\left(1+m^{2} b^{2}\right)^{\lambda}} .
$$

2. $n$-dependence of $\epsilon_{\text {parallel }}^{\mathrm{H}_{2}}(n)$

With $D \gg 1$ and $b \ll 1$, so replacing the summation by the integration, we have

$$
\begin{aligned}
H_{\lambda}(b, n) \approx & K_{\lambda}(b, n)=\int_{0}^{n} d m \frac{2(n-m)}{\left(1+m^{2} b^{2}\right)^{\lambda}} \\
& =2 n^{2}{ }_{2} \mathrm{~F}_{1}\left(\frac{1}{2}, \lambda, \frac{3}{2},-b^{2} n^{2}\right) \\
& +\left\{\frac{1}{b^{2}(\lambda-1)} \frac{1-\left(1+b^{2} n^{2}\right)^{\lambda-1}}{\left(1+b^{2} n^{2}\right)^{\lambda-1}}\right\},
\end{aligned}
$$

where ${ }_{2} \mathrm{~F}_{1}$ is the hypergeometric function. Taking $n \rightarrow \infty$, we have

$$
\left.\frac{1}{n} K_{\lambda}(b, n)\right|_{n \rightarrow \infty}=\frac{\sqrt{\pi} \Gamma\left(\lambda-\frac{1}{2}\right)}{b \Gamma(\lambda)}
$$


i.e.

$$
\begin{aligned}
\epsilon_{\text {parallel }}^{H_{2}}(\infty) \approx & -\frac{1}{D^{6}}\left\{\frac{9 \pi\left(19 C_{\perp \perp}+10 C_{\perp \|}+3 C_{\|\| \|}\right)}{128 b}\right. \\
& \left.-\left(5 C_{\perp \perp}+C_{\|\| \|}\right)\right\} .
\end{aligned}
$$

Because $b \propto D^{-1}$, Eq.(B11) exhibits the leading $D^{-5}$ asymptotics,

$$
\epsilon_{\text {parallel }}^{\text {add }}(\infty) \propto D^{-5}, \quad \text { when } \quad D \gg 1 \text { and } b \ll 1 \text {. }
$$

Since we are interested in the $n$ dependence of $\epsilon_{\text {parallel }}^{H_{2}}(n)$ at fixed $D$ in the intermediate region, firstly we point out that, the $n$-dependence given by the term inside the bracket in $K_{\lambda}(b, n)$ (Eq. (B9)) contributes $n^{-2(\lambda-1)}$ for $\epsilon_{\text {parallel }}^{H_{2}}(n)$ when $n$ is large. It is much faster than for that contributed from ${ }_{2} \mathrm{~F}_{1}\left(\frac{1}{2}, \lambda, \frac{3}{2},-b^{2} n^{2}\right)(\lambda \geq 3)$. The hypergeometric function involves polynomials of $(b n)^{2}$ and can be generally expressed as the following form:

$$
{ }_{2} \mathrm{~F}_{1}\left(\frac{1}{2}, \lambda, \frac{3}{2},-b^{2} n^{2}\right) \quad=\quad \frac{\sum_{k=0}^{\lambda-2} c_{k}(b n)^{2 k}}{\left(1+(b n)^{2}\right)^{\lambda-1}}+\frac{c_{\lambda} \tan ^{-1}(b n)}{b n},
$$

where $c_{k}$ and $c_{\lambda}$ are coefficients. When $n$ is large, we keep the dominating term in the polynomials, i.e.

$$
{ }_{2} \mathrm{~F}_{1}\left(\frac{1}{2}, \lambda, \frac{3}{2},-b^{2} n^{2}\right) \approx \frac{c_{\lambda-2}(b n)^{2(\lambda-2)}}{\left(1+(b n)^{2}\right)^{\lambda-1}}+\frac{c_{\lambda} \tan ^{-1}(b n)}{b n},
$$

so that

$$
\frac{K_{\lambda}(b, n)}{n} \quad \approx \quad \frac{2 c_{\lambda-2} n(b n)^{2(\lambda-2)}}{\left(1+(b n)^{2}\right)^{\lambda-1}}+\frac{2 c_{\lambda} \tan ^{-1}(b n)}{b} .
$$

Each $K_{\lambda}(b, n) / n$ gives roughly the $n^{-1}$ dependence plus a constant of $\frac{\pi}{2}$ coming from the large $n$ limit of $\tan ^{-1}(b n)^{48}$. However, for our present studies with the hydrogen chain length from $n=2 \sim 25$, the effect of $n$-dependence from $\tan ^{-1}(b n)$ should not be ignored. Because the three terms in $\epsilon_{\text {parallel }}^{H_{2}}(n)$ from $K_{\lambda}(\lambda=3,4,5)$ give the same order of magnitude contributions, it then causes difficulty in seeking proper fitting function for parallel geometry. However, here we aim for qualitatively describing the $n$-dependence behavior for insulating cases. Therefore, the fitting function used for DFT-SAPT result is taken to be of the following form:

$$
\epsilon_{\text {parallel }}^{\text {DFT-SAPT, fit }}(n)=A+B \frac{n^{p}}{\left(1+(b n)^{2}\right)^{2}}+C \tan ^{-1}(b n)
$$


i.e. we further leave the contributions from $K_{4}$ and $K_{5}$ aside. With this fitting function, the

saturation of $\epsilon_{\text {parallel }}^{S A P T, f i t}(n)$ at large $n$ goes as $A+\frac{\pi}{2} C$. As the exponent $p$ approaches 3 for large $\kappa$, the electrons are mostly localized on $\mathrm{H}_{2}$ so that the formation of the chain consisting of isolated $\mathrm{H}_{2}$ could be a reasonable approximation.

\section{REFERENCES}

${ }^{1}$ S. Lebègue, J. Harl, T. Gould, J. G. Ángyán, G. Kresse, and J. F. Dobson, Phys. Rev. Lett. 105, 196401 (2010).

${ }^{2}$ A. Marini, P. García-González, and A. Rubio, Phys. Rev. Lett. 96, 136404 (2006).

${ }^{3}$ J. E. Fischer, H. Dai, A. Thess, R. Lee, N. M. Hanjani, D. L. Dehaas, and R. E. Smalley, Phys. Rev. B 55, R4921 (1997).

${ }^{4}$ P. Leyton, J. S. Gomez-Jeria, S. Sanchez-Cortes, C. Domingo, and M. Campos-Vallette, J. Phys. Chem. B 110, 6470 (2006).

${ }^{5}$ U. D. Venkateswaran, A. M. Rao, E. Richter, M. Menon, A. Rinzler, R. E. Smalley, and P. C. Eklund, Phys. Rev. B 59, 10928 (1999).

${ }^{6}$ K. Autumn, Y. A. Liang, S. T. Hsieh, W. Zesch, W. P. Chan, T. W. Kenny, R. Fearing, and R. J. Full, Nature 405, 681 (2000).

${ }^{7}$ P. Pyykko, Angew. Chem. Int. Edit. 43, 1521 (2004).

${ }^{8}$ H. Schmidbaur and A. Schier, Chem. Soc. Rev. 37, 1931 (2008).

${ }^{9}$ J. F. Dobson, A. White, and A. Rubio, Phys. Rev. Lett. 96, 073201 (2006).

${ }^{10}$ H.-Y. Kim, J. O. Sofo, D. Velegol, M. W. Cole, and A. A. Lucas, J. Chem. Phys. 124, 074504 (2006).

${ }^{11}$ D. B. Chang, R. L. Cooper, J. E. Drummond, and A. C. Young, Phys. Lett. A 37, 311 (1971).

${ }^{12}$ N. D. Drummond and R. J. Needs, Phys. Rev. Lett. 99, 166401 (2007).

${ }^{13}$ A. J. Misquitta, J. Spencer, A. J. Stone, and A. Alavi, Phys. Rev. B 82, 075312 (2010).

${ }^{14}$ T. Gould, E. Gray, and J. F. Dobson, Phys. Rev. B 79, 113402 (2009).

${ }^{15}$ T. Gould, K. Simpkins, and J. F. Dobson, Phys. Rev. B 77, 165134 (2008).

${ }^{16}$ C. Hättig, G. Jansen, B. A. Hess, and J. G. Ángyán, Mol. Phys. 91, 145 (1997).

${ }^{17}$ A. J. Misquitta and A. J. Stone, J. Chem. Phys. 124, 024111 (2006). 
${ }^{18}$ G. J. Williams and A. J. Stone, J. Chem. Phys. 119, 4620 (2003).

${ }^{19}$ A. J. Stone, Mol. Phys. 56, 1065 (1985).

${ }^{20}$ J. G. Ángyán, G. Jansen, M. Loss, C. Hättig, and B. A. Hess, Chem. Phys. Lett. 219, 267 (1994).

${ }^{21}$ J. G. Ángyán, J. Chem. Phys. 127, 024108 (2007).

${ }^{22}$ H. C. Longuet-Higgins and L. Salem, Proc. Roy. Soc. A 259, 433 (1961).

${ }^{23}$ A. White and J. F. Dobson, Phys. Rev. B 77, 075436 (2008).

${ }^{24}$ B. Champagne, D. H. Mosley, M. Vračko, and J.-M. André, Phys. Rev. A 52, 178 (1995).

${ }^{25}$ S. Kümmel, L. Kronik, and J. P. Perdew, Phys. Rev. Lett. 93, 213002 (2004).

${ }^{26}$ Z. Medin and D. Lai, Phys. Rev. A 74, 062508 (2006).

${ }^{27}$ A. V. Sinitskiy, L. Greenman, and D. A. Mazziotti, J. Chem. Phys. 133, 014104 (2010).

${ }^{28}$ T. Tsuchimochi and G. E. Scuseria, J. Chem. Phys. 131, 121102 (2009).

${ }^{29}$ M. van Faassen, P. L. de Boeij, R. van Leeuwen, J. A. Berger, and J. G. Snijders, Phys. Rev. Lett. 88, 186401 (2002).

${ }^{30}$ A. Heßelmann and G. Jansen, Chem. Phys. Lett. 357, 464 (2002).

${ }^{31}$ A. Heßelmann and G. Jansen, Chem. Phys. Lett. 362, 319 (2002).

${ }^{32}$ A. Heßelmann and G. Jansen, Phys. Chem. Chem. Phys. 5, 5010 (2003).

${ }^{33}$ A. Heßelmann and G. Jansen, Chem. Phys. Lett. 367, 778 (2003).

${ }^{34}$ G. Jansen and A. Heßelmann, J. Phys. Chem. A 105, 11156 (2001).

${ }^{35}$ A. J. Misquitta, B. Jeziorski, and K. Szalewicz, Phys. Rev. Lett. 91, 033201 (2003).

${ }^{36}$ A. J. Misquitta, R. Podeszwa, B. Jeziorski, and K. Szalewicz, J. Chem. Phys. 123, 214103 (2005).

${ }^{37}$ H.-J. Werner, P. J. Knowles, F. R. Manby, M. Schütz, P. Celani, G. Knizia, T. Korona, R. Lindh, A. Mitrushenkov, G. Rauhut, et al. (2008), see http://www.molpro.net.

${ }^{38}$ A. Karpfen, Chem. Phys. Lett. 61, 363 (1979).

${ }^{39}$ C.-M. Liegener, J. Phys. C: Solid State Phys. 18, 6011 (1985).

${ }^{40}$ S. Suhai and J. Ladik, J. Phys. C: Solid State Physics 15, 4327 (1982).

${ }^{41}$ J. G. Ángyán, Curr. Org. Chem. (2010), in press.

${ }^{42}$ R. Resta, J. Phys.: Condens. Matter 14, R625 (2002).

${ }^{43}$ R. Resta, J. Chem. Phys. 124, 104104 (2006).

${ }^{44}$ R. Resta and S. Sorella, Phys. Rev. Lett. 82, 370 (1999).

${ }^{45}$ A. G. De Rocco and W. G. Hoover, Proc. Natl. Acad. Sci. U. S. 46, 1057 (1960). 
${ }^{46} \mathrm{~B}$. Noble, Methods based on the Wiener-Hopf technique for the solution of partial differential equations, American Mathematical Society (1988).

${ }^{47}$ N. Wiener and E. Hopf, Über eine Klasse singulärer Integralgleichungen, Sitzungber. Akad. Wiss. Berlin (1931).

${ }^{48}$ L. Salem, J. Chem. Phys. 37, 2100 (1962).

${ }^{49}$ R. Zwanzig, J. Chem. Phys. 39, 2251 (1963). 


\section{FIGURES}




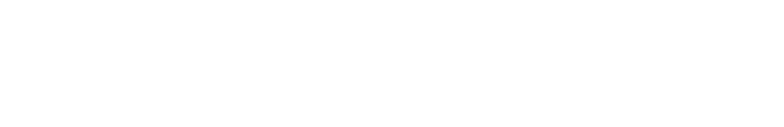

(a)

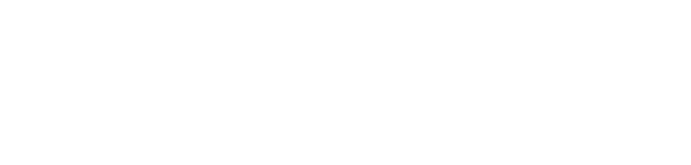

(b)

FIG. 1. Geometry configurations: a) two pointing chains, b) two parallel chains. $r=1.84 a_{0}$ and $D=8 a_{0}$. 


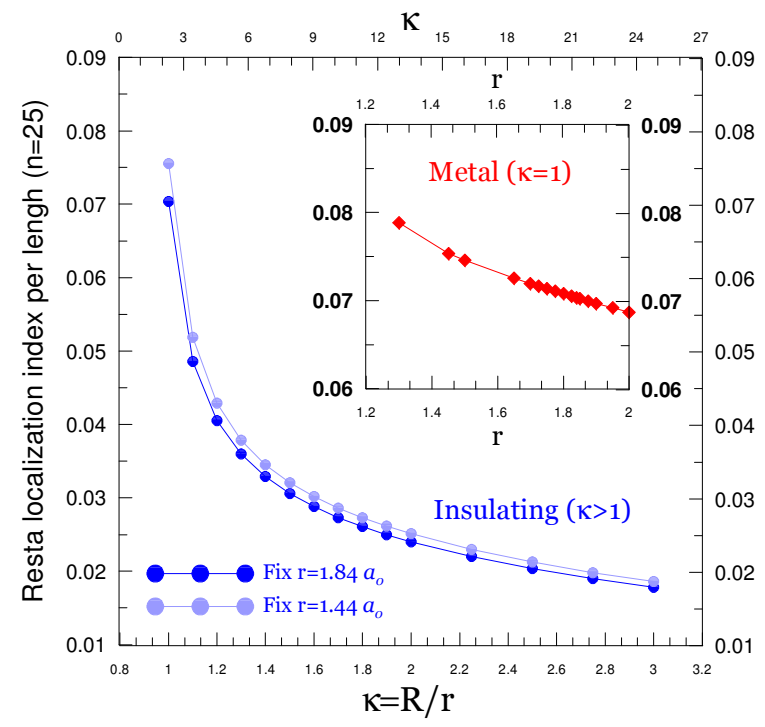

FIG. 2. Resta localization index $\left\langle r^{2}\right\rangle_{c}$ per length evaluated by using exchange hole for the hydrogen chain with $n=25$. 


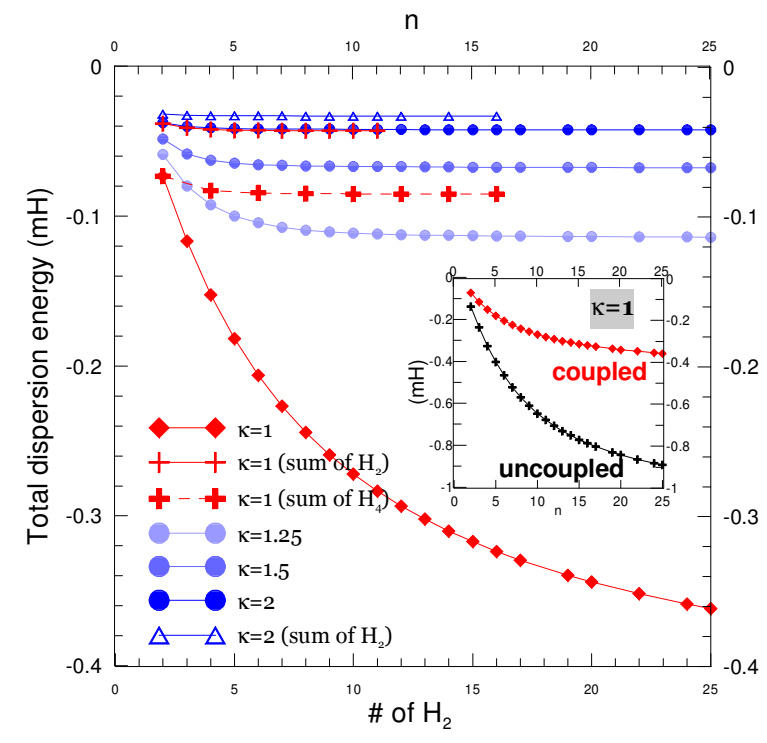

FIG. 3. Total dispersion energy $(\mathrm{mH})$ for pointing geometry. 


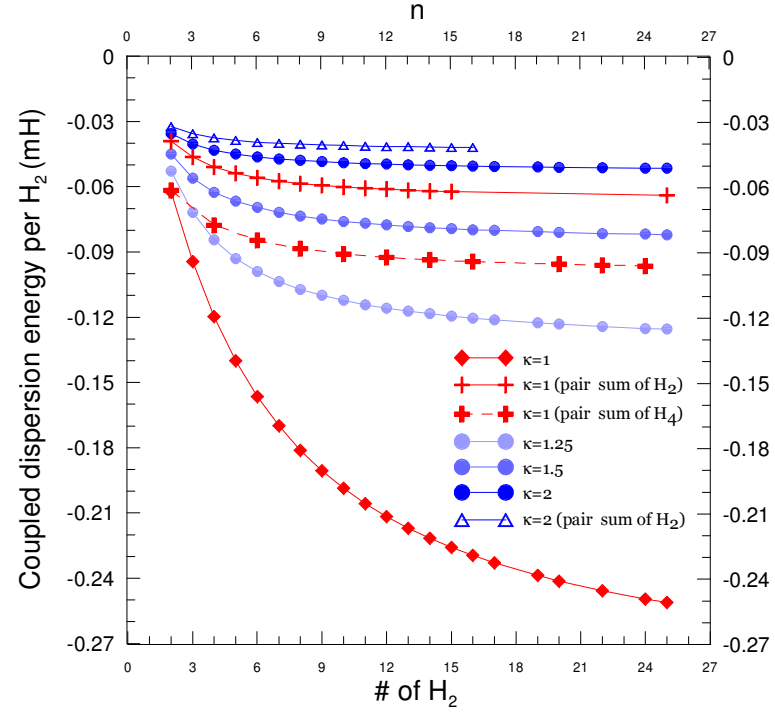

FIG. 4. Dispersion energy per $n(\mathrm{mH})$ for parallel geometry. 
TABLES 
TABLE I. HOMO-LUMO gaps (in eV).

\begin{tabular}{lcccc}
\hline \hline $\mathrm{n}$ & $\kappa=1$ & 1.25 & 1.5 & 2 \\
\hline 2 & 0.309 & 0.333 & 0.352 & 0.376 \\
3 & 0.243 & 0.281 & 0.312 & 0.352 \\
4 & 0.251 & 0.290 & 0.340 \\
5 & 0.201 & 0.231 & 0.276 & 0.332 \\
10 & 0.172 & 0.187 & 0.248 & 0.319 \\
15 & 0.103 & 0.172 & 0.240 & 0.316 \\
20 & 0.075 & 0.166 & 0.236 & 0.315 \\
25 & 0.060 & 0.162 & 0.235 & 0.314 \\
\hline \hline
\end{tabular}


TABLE II. Numerical fits of $E_{\text {disp }}^{c}$ for pointing geometry.

\begin{tabular}{cccc}
\hline \hline$\kappa$ & $A$ & $B$ & $p$ \\
\hline $1^{\mathrm{a}}$ & -0.417 & 1.163 & 1.282 \\
1.25 & -0.117 & 1.986 & 2.339 \\
1.5 & -0.067 & 3.682 & 3.655 \\
2 & -0.042 & 0.791 & 3.833 \\
\hline \hline
\end{tabular}

a fit to the function of Eq.(6) 
TABLE III. Numerical fits of $\epsilon_{d i s p}^{c}$ for parallel geometry.

\begin{tabular}{cccccr}
\hline \hline$\kappa$ & $\mathrm{A}$ & $\mathrm{B}$ & $\mathrm{P}$ & $\mathrm{C}$ & $\epsilon^{\text {fit }}(\infty)^{\mathrm{a}}$ \\
\hline $1^{\mathrm{b}}$ & -0.297 & 0.673 & 1.196 & - & -0.297 \\
1.25 & 0.047 & -0.018 & 0.848 & -0.116 & -0.134 \\
1.5 & 0.010 & -0.008 & 1.314 & -0.061 & -0.086 \\
2 & 0.003 & -0.004 & 2.612 & -0.036 & -0.053 \\
\hline \hline a given by $A+\frac{\pi}{2} C$ & & &
\end{tabular}

given by $A+\frac{\pi}{2} C$
b fit to the function of Eq. $(6)$ 